\section{Mieloma múltiple en Chile: Respuesta a tratamiento en pacientes con mieloma múltiple elegibles para trasplante autólogo de progenitores hematopoyéticos}

\author{
CAMILA PEÑA ${ }^{1}$, JORGE ROJAS-VALLEJOS ${ }^{2, \mathrm{a}, \mathrm{b}}$, \\ MARCELA ESPINOZA ${ }^{3}$, JAVIERA DONOSO $^{4}, \mathrm{PABLO} \mathrm{SOTO}^{5}$, \\ DANIELA CARDEMIL ${ }^{6}$, SANDRA ARANDA ${ }^{7}$, \\ CAROLINA CONTRERAS ${ }^{8}$, CARMEN GLORIA VERGARA $^{9}$, \\ GABRIEL LA ROCCA ${ }^{10}$, ROCÍO OSORIO ${ }^{11}$, HERNÁN LÓPEZ-VIDAL ${ }^{12,15}$, \\ MAURICIO CHANDÍA ${ }^{13}$, CHRISTINE ROJAS ${ }^{14}$
}

\section{Response rates to first-line treatment in eligible patients to autologous stem transplantation in Chile}

Background: The treatment of choice of newly diagnosed multiple myeloma (NDMM) is an induction with proteasome inhibitors followed autologous stem cell transplantation (HSCT). Since 2013, the treatment of these patients in the public system is based on CTD (cyclophosphamide, thalidomide, and dexamethasone). Aim: To evaluate the response rates achieved with CTD, and the results of HSCT in patients with NDMM in the public setting. Material and Methods: Data from patients considered as candidates for HSCT from different centers of the National Adult Antineoplastic Drug Program (PANDA, for its acronym in Spanish), diagnosed between 2013 and 2017, was analyzed. The response to treatment of first and second lines of treatment was evaluated, in addition to the results of HSCT. An optimal Response was defined as the sum of strict complete remission, complete remission and very good partial response (sCR, CR and VGPR). Results: One hundred and seventy-seven patients were analyzed, 54\% women, and 53\% with IgG multiple myeloma. Information about the international staging system was retrieved in 127 patients (71\%). Seventeen percent were ISS I, 22\% in ISS II and 32\% ISS III. CTD was used as first treatment in 106 patients (60\%), and cyclophosphamide, bortezomib and dexamethasone (CyBorD) in 13 (7\%). As first line, CTD had an overall response of $50.9 \%$, and CyBorD of $76.9 \%$. Thirty patients were treated with bortezomib as second line treatment. Forty patients (22\%) underwent HSCT. The 5-year Overall Survival (OS) in transplanted patients and non-transplanted patients was 100 and $62 \%$ respectively $(p<0.01)$. Conclusions: The response rate achieved by CTD in these patients is suboptimal. The response to CyBorD was better.

(Rev Med Chile 2019; 147: 1561-1568)

Key words: Bortezomib; Dexamethasone; Hematopoietic Stem Cell Transplantation; Multiple Myeloma.
'Sección de Hematología, Servicio de Medicina. Hospital del Salvador. Santiago, Chile. ${ }^{2}$ Facultad de Economia y Negocios, Universidad Andres Bello. Santiago, Chile. ${ }^{3}$ Escuela de Medicina, Facultad de Ciencias Médicas, Universidad de Santiago de Chile. Santiago, Chile. ${ }^{4}$ Unidad de Hematología. Hospital Sótero del Río. Santiago, Chile.

5Unidad de Hematología, Servicio de Hematología, Oncología y Cuidados Paliativos. Hospital de Puerto Montt. Puerto Montt, Chile.

${ }^{6}$ Servicio de Medicina Interna, Hospital Clínico de Magallanes. Punta Arenas, Chile.

${ }^{7}$ Servicio de Hematología. Hospita San Juan de Dios. Santiago, Chile.

${ }^{8}$ Servicio de Medicina Interna. Hospital Regional de Rancagua.

Rancagua, Chile.

${ }^{9}$ Servicio de Hematología. Hospital Regional de Rancagua. Rancagua, Chile.

${ }^{10}$ Unidad de Hematología. Hospital Regional de Coyhaique. Coyhaique, Chile.

"Sección de Hematología, Servicio de Medicina. Hospital Regional de Arica, "Dr. Juan Noé Crevani" Arica, Chile.

${ }^{12}$ Servicio de Hematología. Hospital Barros Luco-Trudeau. Santiago, Chile.

${ }^{13}$ Servicio de Hematología. Hospital Regional de Concepción. Concepción, Chile.

${ }^{14}$ Unidad de Hematología, Servicio de Medicina. Hospital "Dr.

Gustavo Fricke". Viña del Mar,

Chile.

${ }^{15} \mathrm{Hematología,} \mathrm{Unidad} \mathrm{de}$ Oncología. Clínica Las Condes. Santiago, Chile.

Ingeniero Civil.

bPhD.

Fuente de apoyo financiero: No hay.

Recibido el 11 de septiembre de 2019, aceptado el 13 de diciembre de 2019.

Correspondencia a: Jorge Rojas-Vallejos Facultad de Economía y Negocios, UNAB. Av. Fernández Concha 700 Las Condes, Chile. jorge.rojas.v@unab.cl 
E estándar de tratamiento del mieloma múltiple (MM) consiste en inducción, consolidación con altas dosis de quimioterapia y rescate con trasplante autólogo de progenitores hematopoyéticos (TAPH) y mantención con drogas inmunomoduladoras o inhibidores de proteosoma ${ }^{1}$. A pesar de la introducción de nuevas drogas con mayores tasas de respuesta, que han doblado la sobrevida ${ }^{2}$, en el sistema público de salud de nuestro país aún se están usando esquemas antiguos con vincristina, adriamicina y dexametasona $(\mathrm{VAD})^{3}$, y desde 2013 ciclofosfamida, talidomida y dexametasona $(\mathrm{CTD})^{4}$. Con este último esquema, las tasas de remisión completa (RC) corresponden a $13 \%$ y de muy buena respuesta parcial (MBRP) a $30 \%{ }^{1}$. En algunos casos, y principalmente en segunda línea, algunos centros logran conseguir la compra de bortezomib, usando ciclofosfamida, bortezomib y dexametasona (CyBorD).

Se debe agregar que, si bien pacientes en respuesta parcial también se benefician de $\mathrm{TAPH}^{5}$, solo es posible recibir este procedimiento en el sistema público en pacientes con RC o MBRP, además, de los requisitos de ser paciente de 60 años o menos y sin comorbilidades importantes.

Aquí radica la importancia de evaluar las respuestas obtenidas con el actual régimen de tratamiento en pacientes recientemente diagnosticados de MM en el sistema público.

\section{Material y Métodos}

Estudio retrospectivo observacional, multicéntrico y nacional. Pacientes de 18 a 60 años con MM sintomático recientemente diagnosticado, tratados en el sistema de salud público chileno, entre los años 2013 y 2017. Se analizaron características demográficas, clínicas y terapéuticas. Se definió la respuesta según los criterios del International Myeloma Working Group ${ }^{6}$. Se evaluó respuesta a tratamiento de primera y segunda línea, además de la realización de TAPH. Se consideró en respuesta óptima (RO) a los pacientes en RCs (respuesta completa estricta), RC o MBRP. Se definió respuesta global (RG) a pacientes con $\mathrm{RO}$ o respuesta parcial (RP). Los pacientes se catalogaron según número de líneas de tratamiento: Primera línea como tratamiento inicial; si no lograron MBRP, recibieron segunda y tercera línea de tratamiento, con el objetivo de lograr respuesta y trasplantarse.
Se evaluó sobrevida global (SG) por curvas de Kaplan-Meier y se comparó grupos mediante log rank. Como control de robusticidad se realizó un modelo logístico para analizar las variaciones en la probabilidad de sobrevida dependiendo de las características específicas más relevantes de los pacientes, y así poder verificar los resultados observados en las curvas de sobrevivencia.

\section{Modelo logístico}

Los modelos logísticos se utilizan para analizar la relación que puede existir entre una variable dependiente discreta y un conjunto de variables que pueden ser continuas o discretas ${ }^{6}$. En este caso, la variable dependiente corresponde a si un paciente muere $(Y=1)$ o si sobrevive $(Y=0)$. Dado que esta es una variable dicotómica, entonces aplicamos un modelo logístico binario del tipo,

donde corresponde al conjunto de variables dado por el International Staging System (ISS), CyBorD (que es una variable dummy igual a 1, si el paciente alguna vez recibió CyBorD y 0 si no), sexo (dummy igual a 1 si es mujer y 0 si no) y la edad a la fecha del diagnóstico.

\section{Resultados}

Se recopilaron datos de 177 pacientes candidatos a TAPH durante el período de estudio. El 54\% fueron mujeres. La mediana de edad fue de 52,5 años. Los tipos de MM se dividieron en MM IgG en 53\%, MM IgA en $13,5 \%$ y MM de cadenas livianas (CL) en $21 \%$. Se obtuvo ISS en 127 pacientes $(71 \%)$. Se diagnosticó en ISS I a $17 \%$, en ISS II a $22 \%$ y en ISS III a $32,2 \%$. Las características basales de los pacientes se muestran en la Tabla 1.

Como primera línea se usó CTD en 106 pacientes (59\%), TalDex (talidomida y dexametasona) en $11(6,2 \%)$, CyBorD (ciclofosfamida, bortezomib y dexametasona) en $13(7,3 \%)$ y MPT (melfalán, prednisona y talidomida) en 10 (5,6\%). Los pacientes que recibieron CTD y CyBorD en primera línea obtuvieron RG en 50,9\% y 76,92\%, respectivamente; $y$ de $\mathrm{RO}$ de $30 \%$ y $46 \%$, respectivamente. En segunda línea, con intención de llegar a TAPH, se usó CyBorD en 37 pacientes, los que lograron una $\mathrm{RO}$ en $62 \%$. Las tasas de respuesta global (RG) en la primera línea de tratamiento se muestran en la Tabla 2. 
Tabla 1. Estadísticas descriptivas de la muestra de los pacientes

\begin{tabular}{|lc|}
\hline Variable & $\mathbf{n} / \%$ \\
\hline Edad al diagnóstico [años] & 52,95 \\
Mín-máx [n] & $25-60$ \\
Sexo (mujer = 1) & $96(54 \%)$ \\
ISS & \\
I & $31(17 \%)$ \\
II & $40(22 \%)$ \\
III & $57(32 \%)$ \\
Sin Información & $49(27)$ \\
TAPH & $40(23 \%)$ \\
Tipo de Mieloma & \\
IgA & $24(13 \%)$ \\
IgG & $94(53 \%)$ \\
IgM & $2(1 \%)$ \\
Kappa & $18(10 \%)$ \\
Lambda & $20(11 \%)$ \\
Sin información & $19(10 \%)$ \\
\hline
\end{tabular}

En las Figuras 1, 2 y 3 se muestran las respuestas con la primera, segunda y tercera línea de tratamiento con intención de llegar a TAPH, respectivamente. En segunda línea de tratamiento se muestra una más alta tasa de MBRP, la que después disminuyó en la tercera línea.
Tabla 2. Regímenes quimioterápicos usados en primera línea y tasa de respuesta global

\begin{tabular}{|c|c|c|c|c|}
\hline $\begin{array}{l}\text { Tipo } \\
\text { tratamiento }\end{array}$ & $\mathbf{n}$ & $\begin{array}{c}\text { Frecuencia } \\
{[\%]}\end{array}$ & $\begin{array}{c}\text { Respuesta } \\
\text { global } \\
\text { [n] }\end{array}$ & $\begin{array}{c}\text { Respuesta } \\
\text { global } \\
\text { [frec. \%] }\end{array}$ \\
\hline CTD & 106 & 59,89 & 54 & 50,94 \\
\hline CyBorD & 13 & 7,34 & 10 & 76,92 \\
\hline D & 1 & 0,56 & 1 & 100 \\
\hline MP & 1 & 0,56 & 0 & 0 \\
\hline MPT & 10 & 5,65 & 3 & 30 \\
\hline TD & 11 & 6,21 & 8 & 72,73 \\
\hline VAD & 16 & 9,04 & 11 & 68,75 \\
\hline VD & 1 & 0,56 & 1 & 100 \\
\hline Sin información & 18 & 10,17 & 6 & 33,33 \\
\hline
\end{tabular}

Se realizó TAPH solo en 40 de los pacientes (23\%). De los pacientes que recibieron CyBorD en al menos un ciclo, $34 \%$ se trasplantó vs $17 \%$ en quienes recibieron otros esquemas. La SG a 5 años fue de $70 \%$, con $100 \%$ en pacientes trasplantados versus $62 \%$ en los no trasplantados $(\mathrm{p}=0,0002)$, con sobrevida media de 92 meses versus no alcanzado. Las curvas de sobrevida se muestran en la Figura 4.

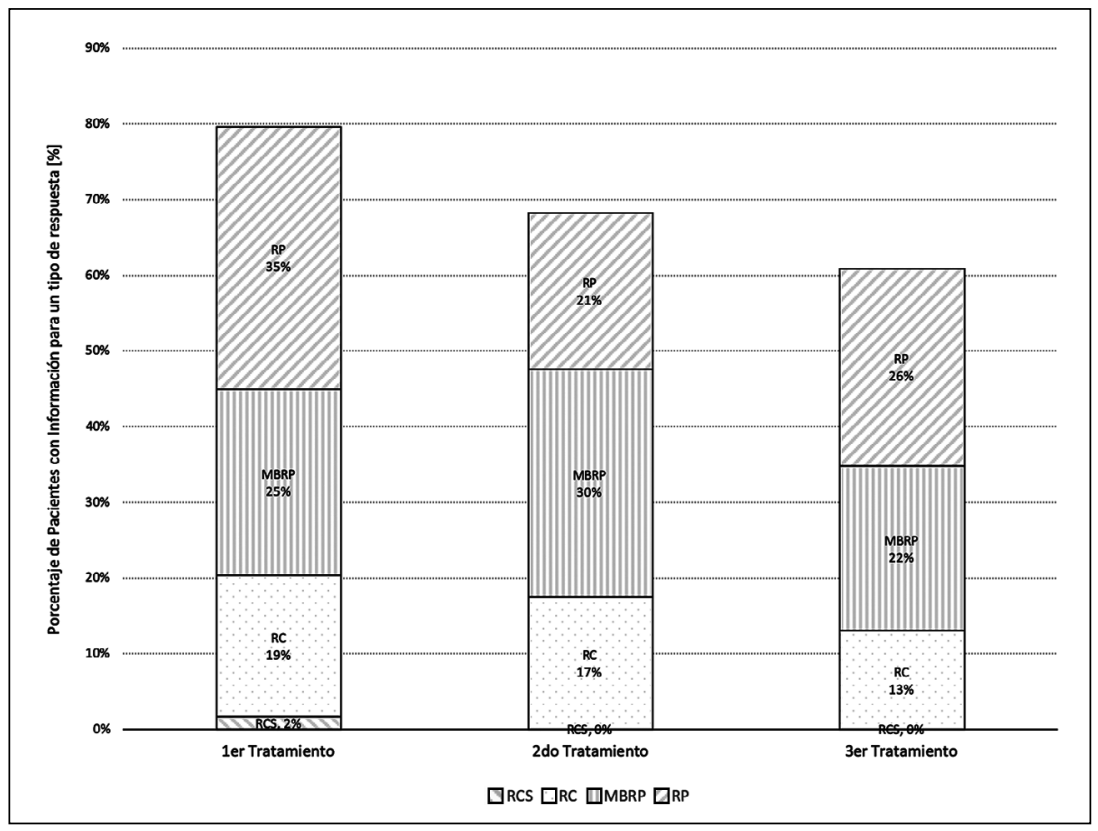

Figura 1. Líneas de tratamiento y criterios de respuesta. 


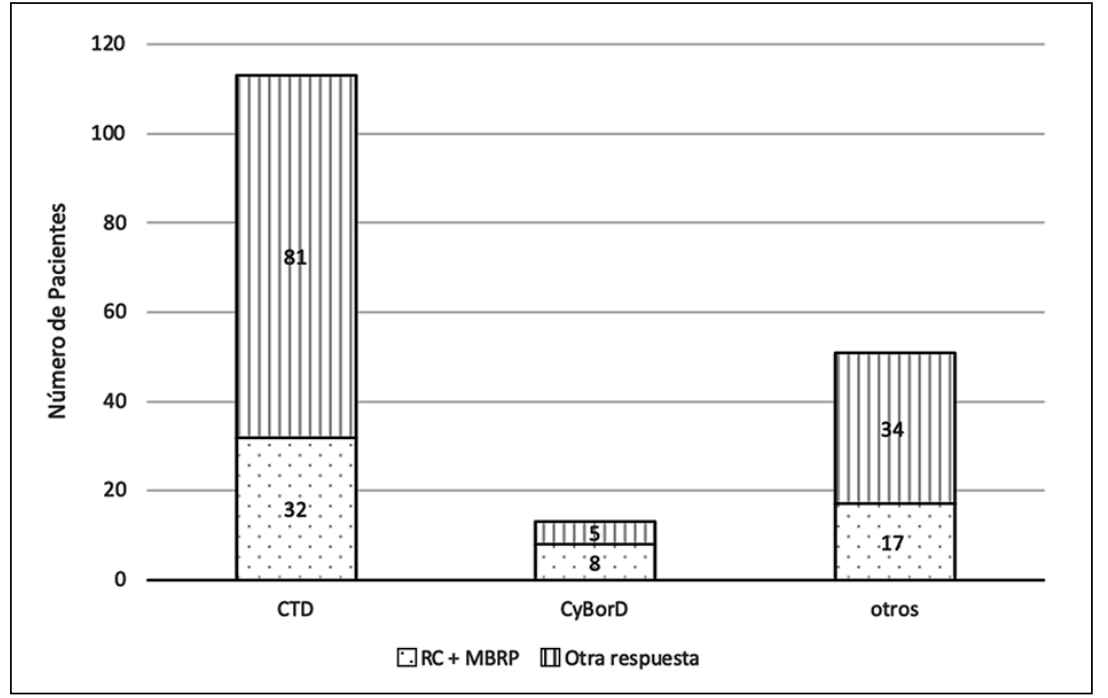

Figura 2. Respuestas a tratamientos. RC: Respuesta completa. MBRP: Muy buena respuesta parcial. RP: Respuesta parcial. CTD: Ciclofosfamida, Talidomida, Dexametasona. CyBorD: Ciclofosfamida, Bortezomib, Dexametasona. RC: Respuesta completa. MBRP: Muy buena respuesta parcial.

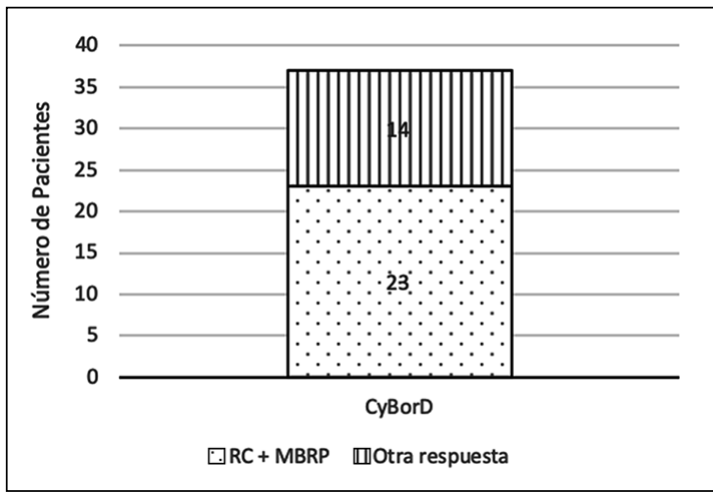

Figura 3. Respuestas a 2a Línea. CyBorD: Ciclofosfamida, Bortezomib, Dexametasona. RC: Respuesta completa. MBRP: Muy buena respuesta parcial.

Utilizando el método de máxima verosimilitud para estimar el modelo logístico y con un tamaño muestral de 128 observaciones disponibles, se encontró que con un nivel de significancia del 1\%, la variable ISS fue estadísticamente significativa en explicar la muerte de un paciente, siendo el efecto marginal de aumentar la probabilidad de muerte aproximadamente $12 \%$. Esto es, para un aumento en el ISS de una unidad, la probabilidad de muerte aumenta en $12 \%$, manteniendo todo lo demás constante. De igual modo, cuando el paciente fue tratado con CyBorD, se encontró al
$5 \%$ de significancia que la probabilidad de muerte disminuyera en $22 \%$, comparado con aquellos pacientes tratados con otras drogas, manteniendo todo lo demás constante (ceteris paribus).

Realizando el mismo tipo de análisis, pero para la probabilidad de trasplante, se obtuvo que las variables con mayor significancia estadística correspondieron a ISS y la edad a la fecha del diagnóstico. Ambas disminuyeron la probabilidad de trasplante a medida que aumentaban. Por cada unidad que aumenta el ISS, la probabilidad de trasplante cayó en 7\%, asumiendo otras variables constantes. Mientras que para la edad, la probabilidad de trasplante cayó en $1 \%$, manteniendo otras variables inalteradas. Si bien el efecto de la edad fue pequeño, de todos modos, fue estadísticamente significativo.

\section{Discusión}

El presente estudio mostró que el régimen usado hasta 2017 (CTD) es insuficiente para llegar a $\mathrm{RO}$ y así lograr trasplantar a los pacientes con MM recientemente diagnosticados en nuestro sistema público de salud.

En cuanto a características demográficas y clínicas, llamó la atención el predominio del sexo femenino. Ya había sido reportado en un estudio chileno publicado ${ }^{8}$. Solo encontramos esta pre- 

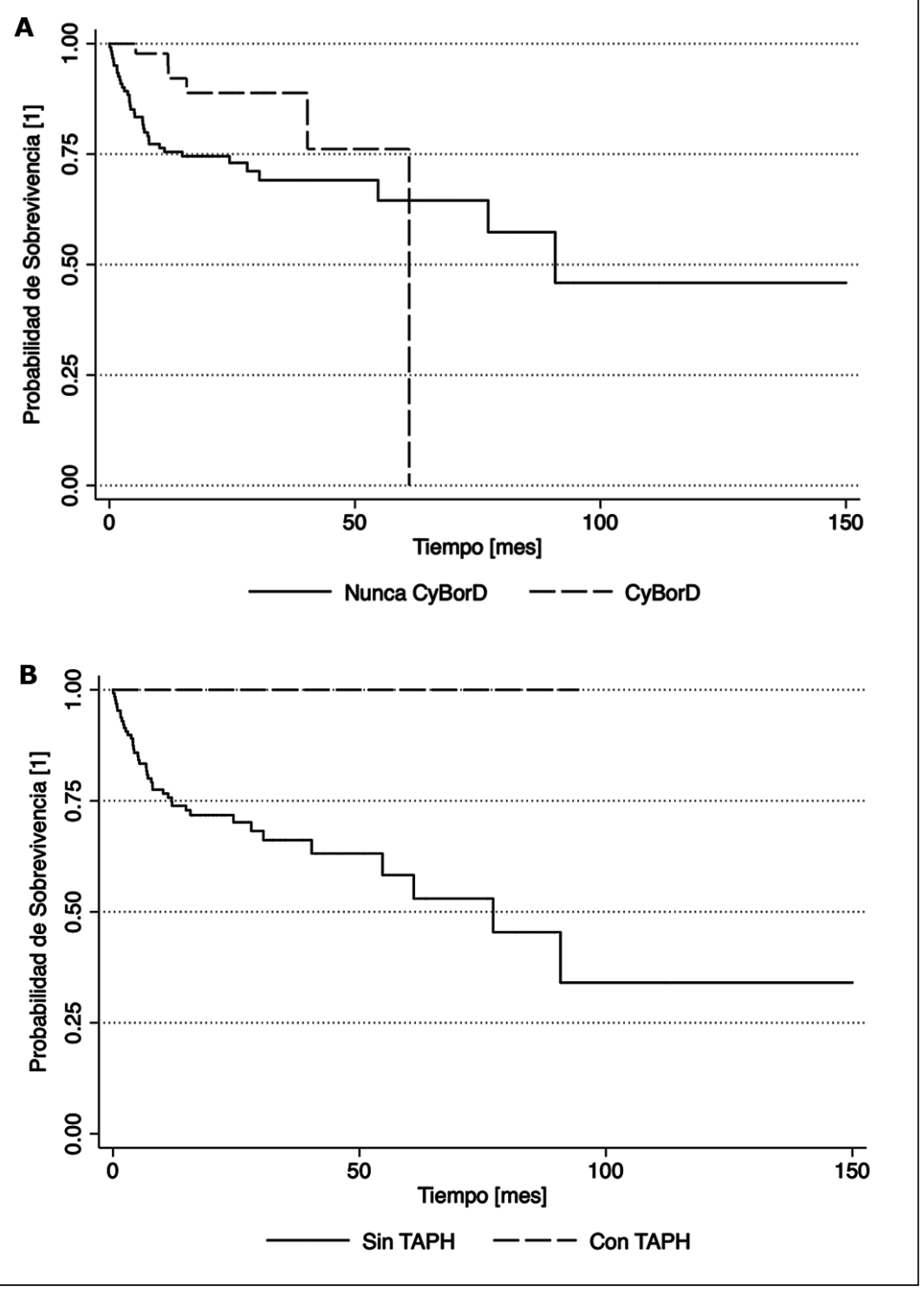

Figura 4. Curvas de sobrevivencia de pacientes tratados con CyBorD y trasplantados. A: Curva de sobrevivencia pacientes que utilizaron CyBorD y los que nunca utilizaron CyBorD $\left(n=42 ; \chi^{2}=3,54\right.$, valor $\mathrm{p}=0,060)$. B: Curva de sobrevivencia Pacientes trasplantados (con TAPH) y los que no ( $\sin$ TAPH $)\left(n=42 ; \chi^{2}=15.64\right.$, valor $p=0,0001)$. dominancia de mujeres en otro reporte en que se caracteriza una cohorte de pacientes brasileños con MM de bajo nivel socioeconómico. Ellos reportaron una relación $\mathrm{H} / \mathrm{M}$ de $0,6 / 1^{9+}$.

La mayor parte de nuestros pacientes candidatos a TAPH fueron diagnosticados en ISS III, lo que es común en Latinoamérica, donde el MM se diagnostica en etapas más avanzadas. Una posible explicación de esto es el menor acceso al sistema de salud en la región. En un estudio sobre pacientes con MM en Latinoamérica, Hungria et al. reportaron $72 \%$ en etapa III de Durie Salmon y $30 \%$ en ISS de III $^{10}$.

Como se mencionó al principio, el régimen recomendado y financiado por el sistema público chileno desde 2013 y hasta la fecha de este estudio es CTD. No existe financiamiento para drogas de nueva generación como lenalidomida, bortezomib o daratumumab, lo que es muy recurrente en la región. En el presente estudio solo 6,2\% logró usar bortezomib como primera línea. En el estudio de Hungria et al. ${ }^{10}$, que estudió a 852 pacientes lati- 
noamericanos, el régimen más usado en primera línea (casi en dos tercios de los pacientes) fue el VAD. El 20\% recibió talidomida y dexametasona; ningún paciente recibió bortezomib o lenalidomida. Es más, menos de $10 \%$ de los candidatos a TAPH los recibió como quimioterapia de rescate. En otro estudio brasileño, que incluyó MM diagnosticados desde 2006 al 2014, el régimen más usado fue el CTD, usado en $50,7 \%$.

En este punto cabe destacar que desde octubre de 2019, el sistema público comenzará a financiar bortezomib y lenalidomida en estos pacientes, a través de la Ley de Garantías Explícitas en salud (GES).

\section{Respuestas globales con CTD}

Como se mencionó, las indicaciones para llevar a TAPH a nuestros pacientes son limitadas. Una de estas limitaciones es que los pacientes deben lograr respuesta equivalente a $\mathrm{RO}$. Nuestros resultados muestran una tasa de RG con CTD de 50,94\%, mientras que la RO llegó a 30\%. Estos resultados son menores comparados con la literatura: Morgan et al. ${ }^{4}$ reportó una RG de $82,5 \%$ y una $\mathrm{RO}$ de $43,2 \%$. Yang et al. ${ }^{11}$ encontró una RG $87,7 \%$ y una RO de $47,7 \%$. Sidra et al. y Wu et al. ${ }^{12,13}$ mostraron RO de $21 \%$ y $45 \%$, respectivamente, con CTD en primera línea.

El bortezomib, fármaco aprobado por la Food And Drug Administration (FDA) de los Estados Unidos de Norteamérica desde el 2008 como primera línea en MM, causa farmacológicamente proteolisis del inhibidor kappa beta, incrementando el factor nuclear kappa beta NFK $b^{14}$. Se describen tasas de RO de 32\%-62\% ${ }^{15-17}$. En nuestro estudio, los pacientes que recibieron inducción con bortezomib fueron la minoría. Sin embargo, la diferencia en respuestas globales alcanzadas fue importante en comparación con CTD (76\% versus $50 \%$ ). Esta mejor respuesta se observa incluso al analizar los pacientes que usaron en segunda línea este fármaco, con mayores tasas de MBRP en este grupo. Esperamos que la introducción de nuevas drogas por parte del GES incremente significativamente las respuestas obtenidas con el actual régimen.

\section{Trasplante}

En general, se trasplanta a un tercio de todos los pacientes con diagnóstico de $\mathrm{MM}$, ya sea por avanzada edad o por comorbilidades importantes.
En la experiencia de la Clínica Mayo desde 2001 al 2010 se realizó TAPH a 37\% del total de pacientes, y un $56 \%$ de los que eran elegibles ${ }^{2}$. En el reporte de Hungria et al. ${ }^{10}$, el TAPH se realizó a $26,9 \%$ de los pacientes, un 58,6\% de los candidatos. Según lo reportado por Peña et al. ${ }^{8}$, en Chile sólo logran trasplante $6,6 \%$ del total de los pacientes con MM, y $18 \%$ de los que se consideran candidatos, una cifra inaceptable. Nuestro reporte va en esa línea, ya que encontramos que sólo $23 \%$ de los candidatos a TAPH en el sistema público chileno reciben finalmente este procedimiento.

Como ya se mencionó, varios factores son los que explican esta baja tasa de trasplante en pacientes considerados candidatos a este procedimiento al diagnóstico. Uno es el criterio de edad, ya que en la mayoría de los protocolos internacionales no existe restricción según este factor. Esto es un importante punto a evaluar, ya que según el modelo de regresión realizado, sólo el ISS y la edad del paciente a la fecha de diagnóstico tienen importancia en determinar si éste podrá llegar al trasplante o no. Por otro lado está la respuesta solicitada (RC o MBRP), que son estrictas considerando que existen estudios que avalan buenas respuestas en pacientes trasplantados en $\mathrm{RP}^{5}$. Si bien las indicaciones para TAPH son estrictas, creemos que además la baja profundidad de las respuestas logradas con CTD u otros regímenes sin bortezomib está influyendo en este mal resultado.

Por otro lado, debemos mencionar que no está garantizada la mantención postrasplante, que se considera manejo estándar ${ }^{18,19}$.

\section{Sobrevida}

En cuanto a sobrevida, Hungria et al. ${ }^{10}$ reportó una SG a 5 años estimada de $56 \%$ con regímenes basados mayormente en talidomida. Otro estudio con inducciones con CTD, Taldex o VAD tuvo SG a 5 años de $55 \%{ }^{20}$.

En general, nuestra cohorte tiene una SG a 5 años que alcanzó $70 \%$, sin embargo, este dato debe tomarse con cautela, debido a que son pacientes muy jóvenes (60 años o menos), no comparable a otros estudios.

Existe, además, gran diferencia en sobrevida entre los que lograron el TAPH versus los que no lo lograron, lo que nos insta a lograr una mejor terapia de inducción.

De especial consideración y preocupación, es lo reportado en un reciente estudio poblacional ${ }^{21} \mathrm{de}$ 
17 países latinoamericanos, en que se vio que entre 1995 a 2013 Chile tuvo la mayor tasa de mortalidad en pacientes con MM, lo que podría deberse a un diagnóstico más tardío y a un tratamiento menos eficaz. De hecho, un ISS bajo al diagnóstico y el tratamiento con CyBorD fueron los factores más relevantes y estadísticamente significativos en disminuir la probabilidad de muerte, pudiendo llegar a $30 \%$ conjuntamente.

\section{Conclusión}

La respuesta óptima alcanzada por CTD en nuestros pacientes es insuficiente. Con la estrategia actual sólo logró TAPH 23\% de los pacientes candidatos. Creemos que estos resultados dan pie para justificar el financiamiento de estas "nuevas drogas" en primera línea para pacientes candidatos a TAPH en el sistema público y así poder darles a los pacientes un tratamiento más eficaz.

\section{Referencias}

1. Attal M, Lauwers-Cances V, Hulin C, Facon T, Caillot $\mathrm{D}$, Escoffre $\mathrm{M}$, et al. Autologous transplantation for multiple myeloma in the era of new drugs: a phase III study of the Intergroupe Francophone du Myelome (IFM/DFCI 2009 Trial). Blood 2015; 126 (23): 391.

2. Kumar SK, Dispenzieri A, Lacy MQ, Gertz MA, Buadi FK, Pandey S, et al. Continued improvement in survival in multiple myeloma: changes in early mortality and outcomes in older patients. Leukemia 2014; 28 (5): 1122-8.

3. Segeren CM, Sonneveld P, Van der Holt B. Vincristine, doxorubicin and dexamethasone (VAD) administered as rapid intravenous infusion for first-line treatment in untreated multiple myeloma. Br J Haematol 1999; 105 (1): 127-30.

4. Morgan GJ, Davies FE, Gregory WM, Bell SE, Szubert AJ, Navarro Coy N, et al. Cyclophosphamide, thalidomide, and dexamethasone as induction therapy for newly diagnosed multiple myeloma patients destined for autologous stem-cell transplantation: MRC Myeloma IX randomized trial results. Haematologica 2012; 97 (3): 442-50.

5. Gertz MA, Dingli D. How we manage autologous stem cell transplantation for patients with multiple myeloma. Blood 2014; 124 (6): 882-90.

6. Kumar S, Paiva B, Anderson KC, Durie B, Landgren
O, Moreau P. International Myeloma Working Group consensus criteria for response and minimal residual disease assessment in multiple myeloma. Lancet Oncol 2016; 17 (8): e328-46.

7. Prentice R. Use of the logistic model in retrospective studies. Biometrics 1976; 32 (3): 599-606.

8. Peña C, Rojas C, Rojas H, Soto P, Cardemil D, Aranda $\mathrm{S}$, et al. Mieloma múltiple en Chile: Pasado, presente y futuro del programa nacional de drogas antineoplásicas (PANDA). Revisión de 1.103 pacientes. Rev Med Chile 2018; 146: 869-75.

9. Minnicelli C, Maciel J, Hassan R, Lemos T. Clinical and epidemiological features of multiple myeloma patients from a low socio-economic region of Brazil. Rev bras hematol hemoter 2015; 37 (5): 354-5.

10. Hungria VT, Maiolino A, Martínez G, Duarte G, Bittencourt R, Peters L, et al. Observational study of multiple myeloma in Latin America. Ann Hematol 2017; 96 (1): 65-72.

11. Yang DH, Kim YK, Sohn SK, Chung JS, Joo YD, Lee $\mathrm{JH}$, et al. Induction treatment with cyclophosphamide, thalidomide, and dexamethasone in newly diagnosed multiple myeloma: A phase II study. Clinical Lymphoma Myeloma and Leukemia 2010; 10 (1): 62-7.

12. Wu P, Davies FE, Horton C, Jenner MW, Krishnan B, Alvares CL, et al. The combination of cyclophosphamide, thalidomide and dexamethasone is an effective alternative to cyclophosphamide - vincristine - doxorubicin - methylprednisolone as induction chemotherapy prior to autologous transplantation for multiple myeloma: a case-matched analysis. Leuk Lymphoma 2006; 47 (11): 2335-8.

13. Sidra G, Williams CD, Russell NH, Zaman S, Myers B, Byrne JL. Combination chemotherapy with cyclophosphamide, thalidomide and dexamethasone for patients with refractory, newly diagnosed or relapsed myeloma. Haematologica 2006; 91 (6): 862-3.

14. Lü S, Wang J. The resistance mechanism of proteasome inhibitor bortezomib. Biomark Res 2013; 1 (1): 13.

15. Cavo M, Tacchetti P, Patriarca F, Petrucci MT, Pantani L, Galli M, et al. Bortezomib with thalidomide plus dexamethasone compared with thalidomide plus dexamethasone as induction therapy before, and consolidation therapy after, double autologous stem-cell transplantation in newly diagnosed multiple myeloma: a randomised phase 3 study. Lancet 2010; 376 (9758): 2075-85.

16. Palumbo A, Gay F, Falco P, Crippa C, Montefusco V, Patriarca F, et al. Bortezomib as induction before autologous transplantation, followed by lenalidomide as consolidation-maintenance in untreated multiple 
myeloma patients. J Clin Oncol 2010; 28 (5): 800-7.

17. Harousseau JL, Attal M, Avet-Loiseau H, Marit G, Caillot D, Mohty M, et al. Bortezomib plus dexamethasone is superior to vincristine plus doxorubicin plus dexamethasone as induction treatment prior to autologous stem-cell transplantation in newly diagnosed multiple myeloma: results of the IFM 2005-01 phase III trial. J Clin Oncol 2010; 28 (30): 4621-9.

18. Palumbo A, Cavallo F, Gay F, Di Raimondo F, Ben Yehuda D, et al. Autologous transplantation and maintenance therapy in multiple myeloma. N Engl J Med 2014; 371 (10): 895-905.
19. Attal M, Lauwers-Cances V, Marit G, Caillot D, Moreau $\mathrm{P}$, Facon $\mathrm{T}$, et al. Lenalidomide maintenance after stem-cell transplantation for multiple myeloma. N Engl J Med. 2012; 366 (19): 1782-91.

20. Crusoe E, Higashi F, Padilha MP, Miranda EC, Quero A, Almeida $\mathrm{M}$, et al. Outcomes of autologous transplantation for multiple myeloma according to different induction regimens. Rev Bras Hematol Hemoter 2014; 36 (1): 19-24.

21. Curado MP, Oliveira MM, Silva DRM, Souza DLB. Epidemiology of multiple myeloma in 17 Latin American countries: an update. Cancer Med 2018; 7 (5): 2101-8.

Tabla A.1. Modelo logístico probabilidad de muerte

\begin{tabular}{|l|c|c|c|c|c|c|}
\hline Variable & Coef. & Err. Std. & Valor $\mathbf{p}$ & dy/dx & Err. Std. & Valor p \\
\hline ISS & 0,757 & 0,316 & 0,017 & 0,117 & 0,045 & 0,009 \\
\hline CyBorD & $-1,399$ & 0,757 & 0,065 & $-0,217$ & 0,105 & 0,040 \\
\hline Sexo & 0,270 & 0,457 & 0,554 & 0,042 & 0,071 & 0,554 \\
\hline Edad & $-0,020$ & 0,042 & 0,627 & $-0,003$ & 0,006 & 0,621 \\
\hline No Obs. & 128 & \multicolumn{7}{|l}{} \\
\hline RPV & $-62,049$ &
\end{tabular}

Tabla A.2. Modelo logístico probabilidad de trasplante

\begin{tabular}{|l|c|c|c|c|c|c|}
\hline Variable & Coef. & Err. Std. & Valor $\mathbf{p}$ & dy/dx & Err. Std. & Valor $\mathbf{p}$ \\
\hline ISS & $-0,415$ & 0,262 & 0,114 & $-0,074$ & 0,046 & 0,108 \\
\hline CyBorD & 0,578 & 0,463 & 0,211 & 0,103 & 0,081 & 0,206 \\
\hline Sexo & $-0,219$ & 0,443 & 0,622 & $-0,039$ & 0,078 & 0,621 \\
\hline Edad & $-0,068$ & 0,027 & 0,012 & $-0,012$ & 0,005 & 0,013 \\
\hline No Obs. & 128 & \multicolumn{7}{|l}{} \\
\hline RPV & $-65,394$ &
\end{tabular}

RPV corresponde al ratio de pseudoverosimilitud. En ambas tablas se utilizaron errores estándares robustos. Los efectos marginales $(d y / d x)$ se calcularon utilizando el método Delta. 